\title{
UNA PREOCUPACIÓN EN LA ACTUALIDAD: EL CONSUMO DE CANNABIS ASOCIADO A LA ESQUIZOFRENIA
}

\author{
A CURRENT CONCERN: THE CONSUMPTION OF CANNABIS ASSOCIATED WITH SCHIZOPHRENIA \\ Shirley Ponce-Blas',a, Graylin Ruíz-Contreras,a, Luis Medrano-Vega'1,a, Germán Rossani-Alatrista²,b
}

\begin{abstract}
Sr. Editor
La incidencia de la esquizofrenia en los países desarrollados y considerados en vías de desarrollo oscila entre el $7-14 \%$ por cada 100.000 habitantes, según reportes al 2019 de la Organización Mundial de la Salud (OMS)'.

La esquizofrenia es una de las enfermedades mentales más graves que existen, afectando el pensamiento, la voluntad, las emociones y las relaciones sociales del paciente, involucrando de manera directa a sus familiares más allegados ${ }^{2}$.

Por lo general, las causas que llevan al paciente a desarrollar esquizofrenia no se conocen, sin embargo, se sabe que aquellos pacientes donde familiares suyos han padecido esquizofrenia tienen más predisposición a tenerla. Existen corrientes que apoyan fuertemente la teoría que existen alteraciones en el cerebro neonatal, o incluso antes de nacer que van a provocar estas alteraciones estructurales en los neurotransmisores sobre todo en la dopamina y en la serotonina ${ }^{3}$.
\end{abstract}

Se ha observado una relación importante de drogodependencia y esquizofrenia, un ejemplo de ello es lo que sucede en EE. UU. donde se estima que el $40 \%$ de las personas presentaban esta asociación ${ }^{4}$, en Suecia, Nueva Zelanda y Reino Unido el promedio registrado alcanza el $15 \%$ de los pacientes ${ }^{5,6}$.

En el Perú, se calcula que los pacientes con esquizofrenia y antecedentes de consumo de cannabis alcanza el $1.4 \%$ en el ultimo año. Según las evidencias científicas también se ha constatado que el consumo de cannabis se ha asociado en un $15 \%$ con la presencia de ideas delirantes y alucinaciones, asi como trastornos auditivos y/o visuales de carácter transitorio ${ }^{7}$.

En la actualidad se ha observado que la prevalencia de drogodependencia es elevada en los consumidores de cannabis. $Y$ a pesar de lo que se observó inicialmente en algunos estudios sobre el predominio en los varones consumidores de cannabis y la asociación con esquizofrenia, se encontró que la prevalencia esta mas relacionada a las mujeres.

En relación a la edad, existe una prevalencia por encima del $44 \%$ entre el consumo de cannabis y la asociación con esquizofrenia relacionada directamente entre los 20 y 30 años $^{2,7}$. Pero la tendencia por el consumo de cannabis se calcula en un $60 \%$ en pacientes menores de 20 años $^{4}$. (Ver tabla 1)

En estos tiempos donde cada vez es mas frecuente la apertura de leyes para su aceptación como consumo libre en algunos países de Europa y América del Sur; debemos que poner atención en los sectores vulnerables de la población para evitar problemas de salud publica como es el aumento de cuadros de esquizofrenia.

La supervisión de los padres, maestros y profesionales de la salud debe ser una tarea prioritaria para el desarrollo formativo de los niños y facilitar en los adolescentes a entender que el consumo de cannabis puede acarrearles problemas severos que los lleven desarrollar trastornos de la personalidad de manera irreversible.

\footnotetext{
${ }^{1}$ Facultad de Medicina Humana, Universidad Ricardo Palma, Lima-Perú

${ }^{2}$ Instituto de Ciencias Biomedicas, Universidad Ricardo Palma, Lima-Perú.

a Estudiante de medicina humana.

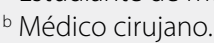

Citar como: Shirley Ponce-Blas, Graylin Ruiz-Contreras, Luis Medrano-Vega, German Rossani-Alatrista. Una preocupación en la actualidad: El consumo de cannabis asociado a la esquizofrenia. Rev. Fac. Med. Hum. Julio 2019; 19(3):107-108. DOI 10.25176/RFMH.v19i3.2150 
Tabla 1. Revisión bibliográfica sobre la prevalencia de esquizofrenia en consumidores de cannabis.

\begin{tabular}{|c|c|c|c|c|c|c|c|c|}
\hline REF. & AÑo & PAÍS & MUESTRA & PREVALENCIA & SEXO & $<20$ AÑ̃os & $\begin{array}{l}\text { EDAD } \\
20 \text { A } 30 \\
\text { AÑOS }\end{array}$ & $>30$ AÑOS \\
\hline 2 & 2012 & Bolivia & 530 & $60 \%$ & $\begin{array}{c}\text { Masculino: } \\
49 \% \\
\text { Femenino: } \\
51 \%\end{array}$ & $23 \%$ & $30 \%$ & $30 \%$ \\
\hline 4 & 2017 & USA & 32330 & $43 \%$ & $\begin{array}{c}\text { Masculino: } \\
33 \% \\
\text { Femenino: } \\
67 \%\end{array}$ & $67 \%$ & $36 \%$ & $1 \%$ \\
\hline 5 & 2004 & $\begin{array}{l}\text { Nueva } \\
\text { Zelanda }\end{array}$ & 759 & $18 \%$ & $\begin{array}{c}\text { Masculino: } \\
59,5 \% \\
\text { Femenino: } \\
40,5 \%\end{array}$ & $65.1 \%$ & $34.1 \%$ & $0.8 \%$ \\
\hline 6 & 2014 & Suecia & 50087 & $17 \%$ & $\begin{array}{c}\text { Masculino: } \\
98 \% \\
\text { Femenino: } \\
2 \%\end{array}$ & $93 \%$ & $4 \%$ & $3 \%$ \\
\hline 7 & 2015 & $\begin{array}{l}\text { Reino } \\
\text { Unido }\end{array}$ & 758 & $9-14 \%$ & $\begin{array}{c}\text { Masculino: } \\
33 \% \\
\text { Femenino: } \\
67 \%\end{array}$ & $19 \%$ & $63 \%$ & $18 \%$ \\
\hline
\end{tabular}

Es importante que el estado implemente nuevas políticas en los sectores de educación, psicología, salud y asistenta social que ayuden en la capacitación en desalentar enérgicamente el consumo de cannabis entre los adolescentes psicológicamente vulnerables.

Nuestra mirada debe estar atenta en la predisposición del sexo femenino como un punto de vulnerabilidad. Sobretodo por la sociedad machista en la que vivimos, donde la mujer esta expuesta a muchos peligros, como la violencia sexual y el feminicidio que nos aqueja en estos últimos años. Lo cual puede desencadenar el uso y el abuso de drogas sobre todo el cannabis.

\section{REFERENCIAS BIBLIOGRÁFICAS}

Contribuciones de autoría: Los autores participaron en la generación, recolección de información, redacción y versión final del artículo original.

Financiamiento: Autofinanciado.

\section{Conflicto de interés: Los autores} declaran no tener conflictos de interés en la publicación de este artículo.

Recibido: 12 de marzo 2019

Aprobado: 12 de mayo 2019

Correspondencia: German Rossani Alatrista.

Dirección: Calle José del Llano Zapata 245, Miraflores, Lima-Perú.

Teléfono: 996414005

Correo: grossani@ingecel.com
1. Organización Mundial de la Salud. Esquizofrenia. Ginebra; 2011.

2. Salguero Jiménez $L$, Soraide J. Factores asociados para el desarrollo de la Esquizofrenia y su incidencia en pacientes del Hospital San Juan de Dios gestión 2012. Rev Cient Cienc Méd. 2012 ; 15(2):18-21.

3. Fonseca-Pedrero E, Lemos-Giraldez S, Paino M, Sierra-Baigrie S, Villazon-Garcia U, Garcia-Portilla Gonzalez M.P, y Muniz J. Dimensionality of hallucinatory predisposition: Confirmatory factor analysis of the Launay-Slade Hallucination Scale Revised in college students. Anales de Psicologia. 2010; 26: 41-48.
4. Vaucher J, Keating B, Lasserre A, Gan W, Lyall D, Ward J, Smith D, Pell J, Sattar N, Pare G, y Holmes, M. Cannabis use and risk of schizophrenia: a Mendelian randomization study. Molecular Psychiatry. 2017; 23(5): 2871292.

6. Manrique-Garcia E, Zammit S, Dalman C, Hemmingsson T, Andreasson $S, y$ Allebeck P. Prognosis of schizophrenia inpersons with and without a history of cannabis use. Psychological Medicine. 2014; 44(12): 2513-2521.

7. Colizzi M, lyegbe C, Powell J, Ursini G, Porcelli A, Bonvino A, et al. Interaction Between Functional Genetic Variation of DRD2 and Cannabis Use on Risk of Psychosis. Schizophrenia Bulletin. 2014; 41(5): 1171-1182. 